\title{
Phacoemulsification and nucleic acid-enriched aerosol: considerations from an eye model of cataract simulation
}

\author{
Andrea Cacciamani ${ }^{1} \cdot$ Fabio Scarinci ${ }^{1} \cdot$ Luca Bruno $^{1} \cdot$ Bijorn Omar Balzamino $^{1}$. Concetta Cafiero ${ }^{2} \cdot$ Agnese $^{3}$. \\ Pamela Cosimi ${ }^{1}$ - Guido Ripandelli ${ }^{1}$ - Alessandra Micera ${ }^{1}$ (i)
}

Received: 5 January 2021 / Revised: 16 July 2021 / Accepted: 27 July 2021 / Published online: 26 August 2021

(c) The Author(s), under exclusive licence to Springer-Verlag GmbH Germany, part of Springer Nature 2021

\begin{abstract}
Purpose The purpose of this study is to investigate whether phacoemulsification can generate aerosolized single-stranded RNA (ssRNA) and retain sequence integrity using an artificial eye model for experimental cataract surgery.

Methods A simulation of cataract surgery was performed using an anterior chamber eye model filled with an ssRNA probe at different scalar dilutions (kanamycin positive control ssRNA). A plastic conical cage was built over the artificial eye surface of the mock-up. A total of 24 tests (twice reproduced) were performed, and five nitrocellulose strips were placed $15 \mathrm{~cm}$ from the artificial surface of the mock-up and used to collect aerosol particles, from each experiment. Phaco-activity was mimicked using a phacoemulsification equipped with a $2.75-\mathrm{mm}$ tip, and strips were removed at the end of the procedure. RNA extraction, reverse transcription, and agarose gel electrophoresis were performed and compared.

Results Strips collected aerosol droplets enriched with ssRNA, mainly at the higher concentrations tested, compared to related untouched standard solutions. Complementary DNA (cDNA) synthesis confirmed the presence of intact ssRNA fragments. As observed from densitometric analysis of resolved RNA in extracted samples and cDNA bands after retrotranscription, lower concentrations of ssRNA were also detected.

Conclusions As the main output of the study, the phaco-generated aerosol can deliver an intact ssRNA sequence. Since the aerosol can potentially reach the operator's face, any biological agent (virus/bacteria) potentially inside the anterior chamber of a patient undergoing cataract surgery, eventually escaping from biomolecular checks, can be potentially infective for operators. The data reported herein suggest that collective versus individual protective countermeasures should always be encouraged in ocular surgery and should not be restricted to coronavirus disease emergencies.
\end{abstract}

Keywords Aerosol $\cdot$ Nucleic acids $\cdot$ Phacoemulsification cataract $\cdot$ Personal protective equipment $\cdot 3 \mathrm{D}$ visual screen $\cdot$ Eye model $\cdot$ COVID-19

Andrea Cacciamani

andrea.cacciamani@fondazionebietti.it

Alessandra Micera

alessandra.micera@fondazionebietti.it

IRCCS-Fondazione Bietti, Rome, Italy

2 Medical Oncology, SG Moscati Hospital, Taranto, Italy

3 CNR-IASI, Catholic University of Sacred Heart, Rome, Italy 


\section{Key messages}

- The presence of biological molecules inside the anterior chamber could occur even in the absence inflammation or in patients with negative tests.

- The coronavirus disease pandemic has significantly influenced both inpatient and outpatient management worldwide

- Aerosol generation can occur in surgical practice representing an important aspect not only for severe acute respiratory syndrome 2 infection but also for other infectious diseases.

\section{Introduction}

A publication storm has been observed regarding studies on the coronavirus disease (COVID-19 pandemic) (March 11, 2020, WHO declaration), particularly focusing on acute respiratory syndrome coronavirus-2 (SARS-CoV-2) features, diagnosis, and therapies [1-3]. Of these studies, some reports highlight the aerosol-associated generating of the SARS-CoV-2 virus, mainly through respiratory droplets from infected subjects, including those, who are asymptomatic [4-6]. Corroborating data indicate that ocular fluids (mainly tears) might represent a "reservoir" for viral replication [7-9]. Some molecular (mainly viral sequences) and antigenic (protein)-based tests have been included in the standard screening procedures (venous blood, dedicated pre/post-surgery swabs, and oropharyngeal/nasopharyngeal swabs) that precede surgery [10-12]. The possibility of a spread of infected 5- $\mu \mathrm{m}$-droplet aerosols $(0.1->100 \mu \mathrm{m}$ in diameter) generated during cataract surgery cannot be excluded [13-15]. Aerosol generation can also occur during vitreoretinal surgery [16]. Due to previous observations implying that aerosols can reach long distances depending on the size of phaco-tips used and that the ophthalmic surgical microscope forces surgeon to come to close distances to the operating field, in the absence of a face shield, an "infected" patient would present a serious risk for operators.

Since corroborating data encompass the "aerosol-generating medical procedure" and the delivery of potentially infected particles, the aim of present study was to verify if phacoemulsification can generate aerosolized single-stranded RNA (ssRNA) retaining its initial sequence in eye models for experimental cataract teaching, which may represent a real source of infection. The outcome of this study was not limited to COVID-19 pandemic.

\section{Methods}

A synthetic eye model was used to test the aerosol produced by phacoemulsification cataract surgery, and conventional reverse transcription PCR analysis was carried out to verify nucleic acid integrity.
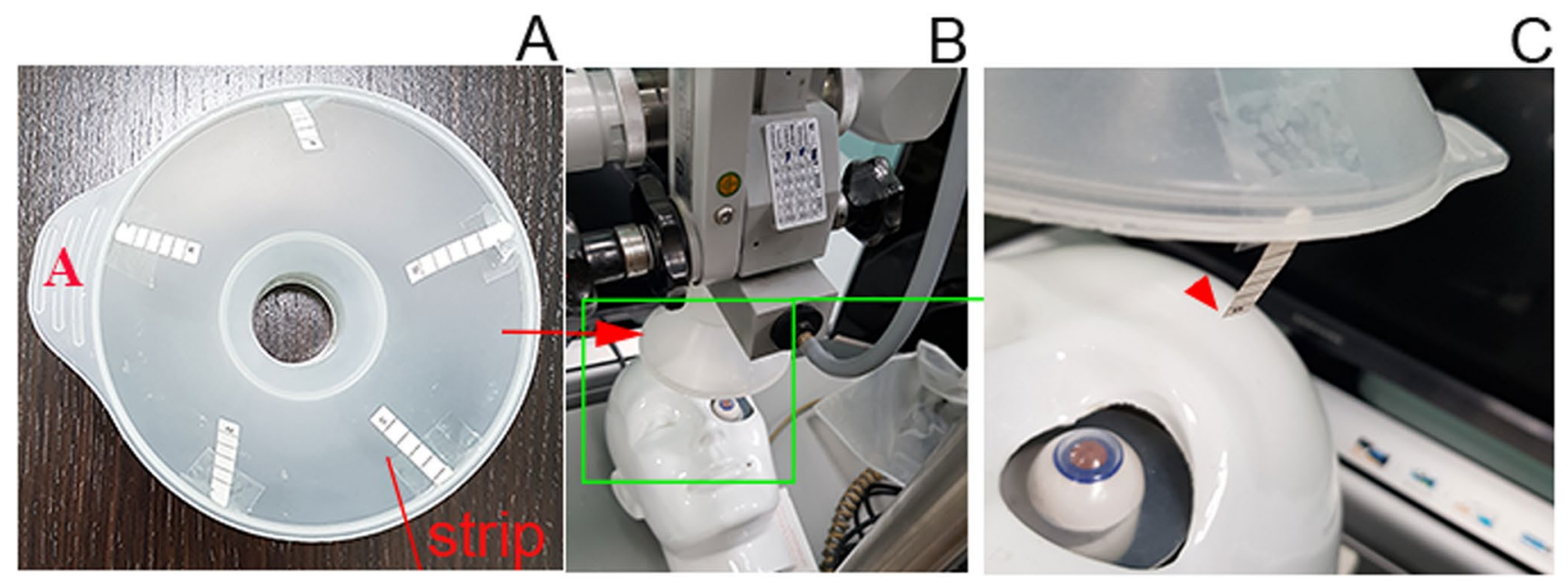

Fig. 1 Description of artificial eye model. The simulation of phacoemulsification for cataract surgery was performed in a wet lab using an eye to investigate the possibility of aerosol transmission (A). Over- view of the surgical training station used for simulation activity (b). Note that the model is placed on a support specific for the training simulation $(\mathbf{C})$. The eye model is framed $(\mathbf{C}$, green square) 


\section{Artificial eye: model set-up and probe}

Five different experimental settings were used for the study, and a total of 42 tests were performed.

The mock-up comprised an artificial anterior segment eye with the possibility of inserting solutions or suspensions inside the anterior chamber. Merely for the study, the chamber was filled with a solution of saline $(400 \mu \mathrm{L})$, alone or supplemented with standard dilutions of an ssRNA probe (5- $\mu \mathrm{L}$ stock solutions for each scalar dilution), through the use of a sterile PIC Insumed syringe $(0.3 \mathrm{~mL} ; 31 \mathrm{G} \times 8 \mathrm{~mm}$; PIC solution). The 1.2-kb kanamycin positive control RNA was used as an ssRNA probe $(10 \mathrm{mM}$ Tris- $\mathrm{HCl}$ and $1 \mathrm{mM}$ EDTA, pH 7.4; Promega, Milan, Italy). The mock-up was placed over an eyeball stand equipped with an optic light and placed under a direct surgery-teaching microscope connected to real-time video for real-time reproduction. As shown in Fig. 1a, a conical cage was equipped with five nitrocellulose strips (Bio-Schirmer, Bio-Tech Vision Care PVT. LTD. Gujarat, India), to allow the collection of aerosol droplets, which eventually aerosolized from the anterior chamber (of mock-up) during phaco-cataract simulation. An overview of the mock-up, including single components, is shown in Fig. 1b. The plastic cage was placed under the objective $15 \mathrm{~cm}$ from the surface of the artificial eye (Fig. 1c).

\section{Experimental phacoemulsification: mimicking procedure and probe collection}

The procedure was carried out in a wet lab developed for teaching purposes (Fig. 1b). A $2.75-\mathrm{mm}$ phacoemulsification tip was used for this study (Alcon, Camberley, Surrey). A wetted artificial surface was obtained using layered BSS, while a dry one was achieved using cellulose eye spears (Weck-Cel, Beaver-Visitec International, Oxford, UK). A dynamic procedure was reproduced by moving the tip inside the artificial anterior chamber to simulate nuclear sculpting, longitudinally along its axis. Before starting each simulation, the conical cage was equipped with a new set of five sterile strips to collect aerosolized droplets for each simulation. Phacoemulsification was reproduced using the Accurus Phaco System (Alcon Inc. Technology, Fort Worth, TX, USA).

Typical phacoemulsification settings were used and the tip was placed in the eye for $1 \mathrm{~min}$ (30\% continuum phacoemulsification power energy, $90-\mathrm{mmHg}$ pressure inside the eye and $250-\mathrm{mmHg}$ vacuum with continuous irrigation). At the end of phacoemulsification simulation, the strips (nitrocellulose Hybond) were quickly removed and hydrated in extraction buffer (lysis buffer: $50 \mathrm{mM}$ Trizma, $300 \mathrm{mM} \mathrm{NaCl}, 20 \mathrm{mg} / \mathrm{mL}$ RNAsin [pH 7.5]; Promega). Probe-containing aerosol droplets were dissolved in the extraction solution (HIRT supplemented with RNAsin) and placed on an orbital shaker (300 rpm for $45 \mathrm{~min}$ ). The strips were quickly sent to the laboratory for specific RNA extraction and quantification.

\section{Molecular analysis: RNA probe extraction and analysis}

RNA was extracted from all test samples using TRIzol reagent (Invitrogen, Milan, Italy) and resuspended in DEPCautoclaved water $(21 \mu \mathrm{L})$. Two-microliter RNA samples were spectrophotometrically analyzed and optical density was recorded according to a standard procedure (A1000 Nanodrop, Celbio, Milan, Italy). Reverse transcription (complementary [cDNA] synthesis) was performed according to a standard procedure (GOScript; Promega). cDNA products were electrophoretically separated on a $2 \%$ agarose gel (Bio-Rad) prepared in TBE (Serva) and supplemented with SYBR green gel stainer $(10 \mu \mathrm{L}$; Molecular Probes). Band separation was carried out at $80 \mathrm{~V}$ with acquisitions at $15 \mathrm{~min}$ and $60 \mathrm{~min}$ after loading. Acquisitions (8-tiff images; $500 \mathrm{dpi}$ ) were carried out using a digital camera (Nikon, Tokyo, Japan) under UV light (Euroclone). Band quantification was carried out using ImageJ software (NIH free-available ImageJ software; https:// imagej.nih.gov/ij/download.html).

\section{Results}

\section{Mock-up and phacoemulsification simulation}

Illustrations of the eye model station with Schirmer strip and artificial anterior chamber were indicated in Fig. 1a, $\mathrm{c}$, respectively. The simulation of phacoemulsification was performed in a wet lab by reproducing the surgical procedure step-by-step. Five experiments were carried out with five scalar dilutions of each probe. At the end of each simulation, strips were removed and used for RNA extraction and followed by reverse transcription to produce $\mathrm{cDNA}$.

\section{Aerosolized ssRNA was collected using strips at the end of experimental phacoemulsification}

A280 analysis showed the presence of ssRNA in strips collected at the end of experimental phacoemulsification. The representative spectral absorbances of untouched scalar dilutions of ssRNA (standard curve; Fig. 2a) and related aerosolized amounts (Fig. 2b) were collected from the strips at the end of the experiment. As expected, the amount of ssRNA was lower in the strips. 
Fig. 2 Probe extraction and cDNA synthesis. Serial singlestranded RNA solutions (dilution factor 2) were introduced into the anterior chamber of the mock-up (400 $\mu \mathrm{L} /$ eye model) and prepared for phacoemulsification experiments. The simulation of phacoemulsification surgery was performed in a wet lab. Spectral absorbance acquisition of standard dilutions (A) and aerosol extracted (B) RNAs; Representative 2\% agarose gel $(\mathbf{C}, \mathbf{E}, \mathbf{G})$ and the related densitometric analysis $(\mathbf{D}, \mathbf{F}, \mathbf{H})$ of resolved complementary DNA products, previously synthesized from the following: the total RNA standard solutions $(\mathbf{C}, \mathbf{D})$, anterior chamber $(\mathbf{E}, \mathbf{F})$, and strip/aerosol (G, H) RNAs. From left to right: M, DNA ladder and 1st-6st (st, standard; c); 1c-6c (c, chamber; D) and $1 \mathrm{~s}-6 \mathrm{~s}$ (s, strip; E). Note the absence of signals at lower concentrations. Densitometric analysis was performed using ImageJ software
A
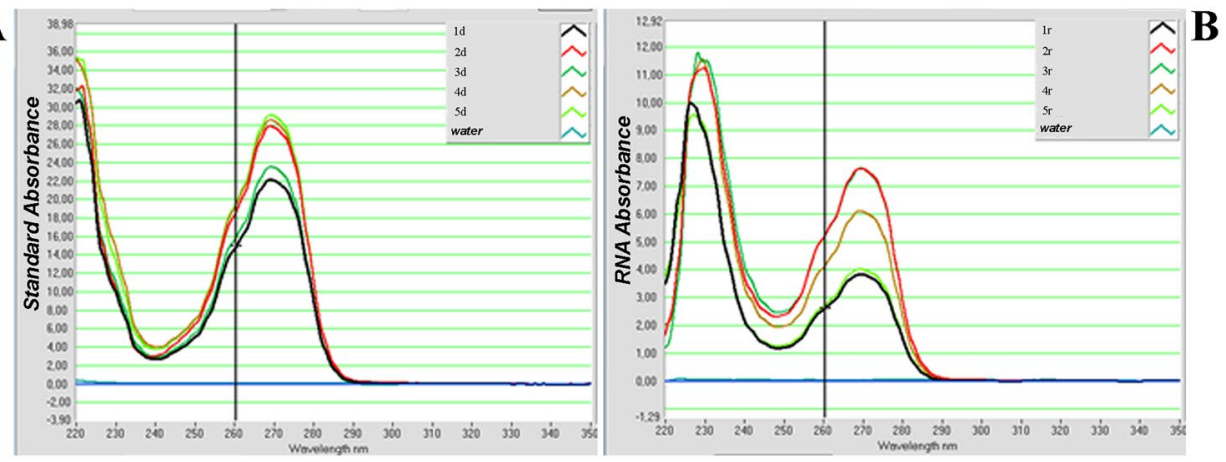

C

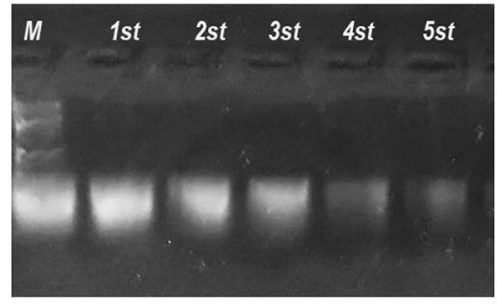

$\mathbf{E}$
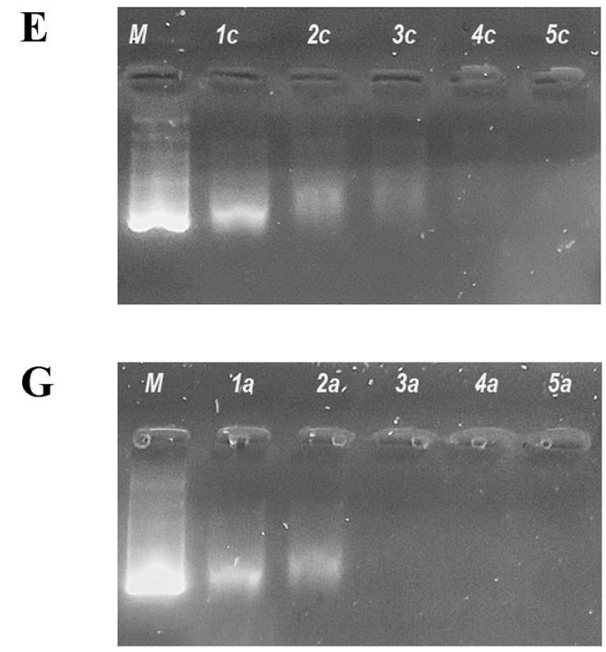

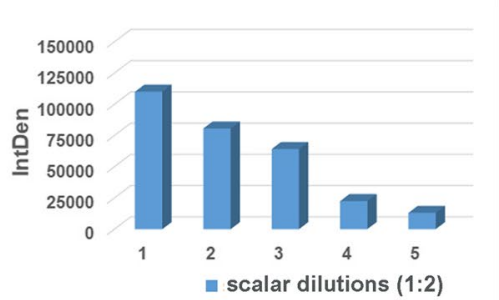

D

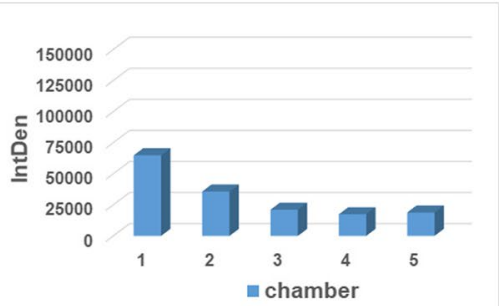

F

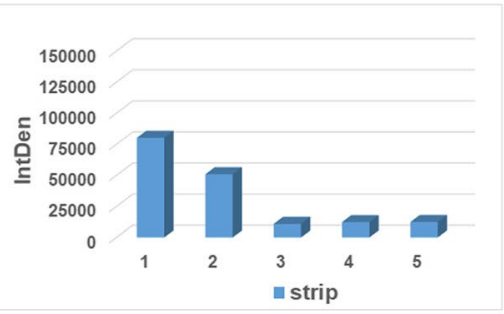

\section{Aerosols did not modify the sequence of ssRNA probes after experimental phacoemulsification}

In order to understand if the phacoemulsification procedure could alter the ssRNA sequence, cDNA synthesis was performed, according to a standard procedure. Representative $2 \%$ agarose gel (Fig. 2c, e, g) and related densitometric analysis (Fig. 2d, f, h) of resolved cDNA products are shown in Fig. 2. The cDNA from standard solutions (Fig. 2c-d), the anterior chamber (Fig. 2e, f), and strip/aerosol (Fig. 2g, h) were used.

As shown, the amount of RT product (cDNA) was lower than that of the related standards and higher than that collected from the artificial chamber (Fig. 2c).

A typical standard curve is shown in Fig. 2c, d. Note the reduced expression of cDNA in samples from the chamber (Fig. 2e, f) and a consistent amount of cDNA at higher concentrations and a quite absent signal in strip samples at lower concentrations (Fig. 2g, h). The probe that aerosolized from phacoemulsification surgery was not concentration-dependent.

\section{Discussion}

The laboratory observations reported herein show that intact aerosol-vehicled ssRNA filaments (as probes in the mock-up simulation) can be aerosolized up to $15 \mathrm{~cm}$ from the source, as quantified in the strips. The "intact" ssRNA filaments on strips were confirmed by reverse transcription (cDNA synthesis) of total ssRNA extracted from the strip compared to those of chamber and untouched standard dilution curves. A positive-sense RNA sequence was chosen as the probe for simulating the SARS-CoV-2 aerosol delivery. 


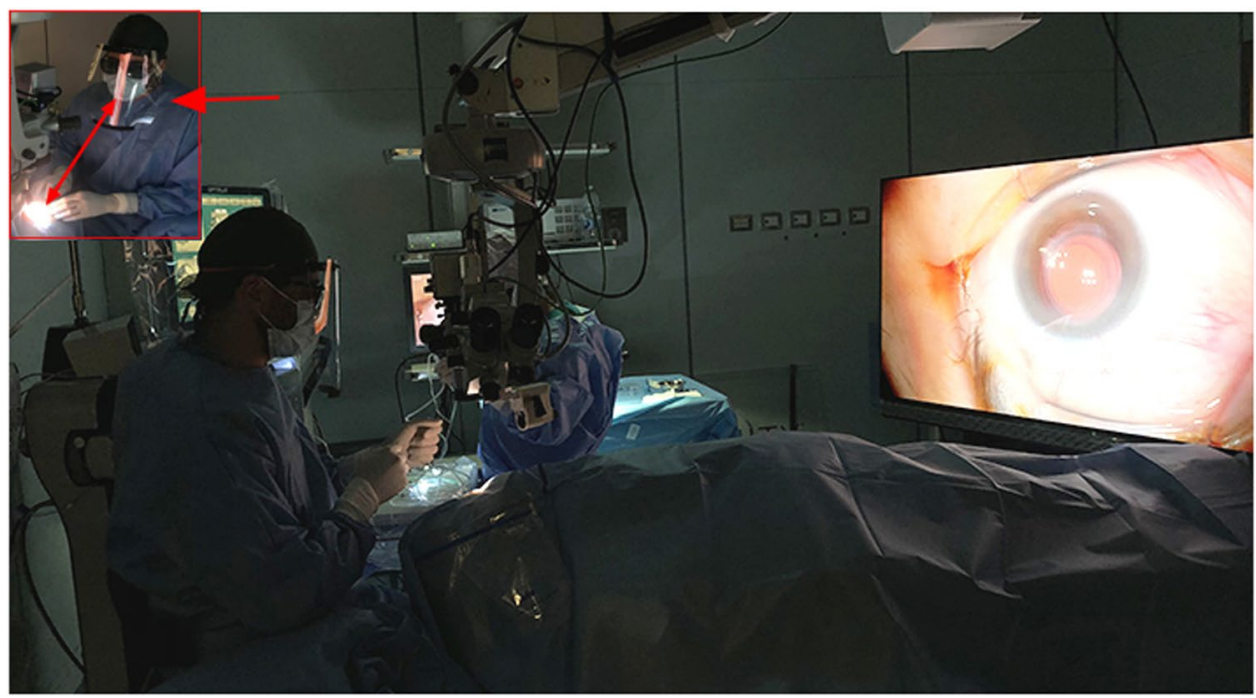

Fig. 3 Representative 3D-assisted surgery session. Picture depicting a phacoemulsification session in the surgery room. Note that the surgeon was wearing a face shield over 3D glasses (see red arrows in upper left insert), while performing the surgery with a 3D-visualization system (NGENUITY®; Alcon Inc. Technology, Fort Worth, TX, USA). The 3D-display unit consists of a camera, a processing unit,

Previous studies suggest that any aerosol droplets can potentially deliver nucleic acids [4-8]. The release of aerosol from experimental phacoemulsification was previously reported using real-time imaging or luminol techniques [17-21]. A porcine-eye mock-up showed that phacoemulsification is a low-risk aerosol-generating procedure, which is also supported by the low transmission risk for SARS-CoV-2 by tears [22-24]. A more recent study highlighted the presence of other techniques for detecting aerosol generated by phacoemulsification (i.e., laser interferometry) and was able to provide clarification of this contrasting literature [13,25]. This latter observation prompted us to further investigate phaco-aerosol generation in experimental models, as the necessity to develop safety strategies is a major concern in ophthalmological practice during this pandemic [26].

Herein, RNA extraction, quantification, and retrotranscription analysis confirmed the delivery and integrity of aerosolized nucleic acid. Probe collections were performed at the end of the phacoemulsification cataract simulation (mock-up), as done in previous studies [22-25]. Comparisons were carried out between the two-factor starting dilutions and the aliquots collected from the chamber-reservoir and Schirmer strips (aerosol captured on a nitrocellulose matrix). The observation of a single undegraded band on agarose gel and the generation of a single band of cDNA products, as synthesized by reverse transcriptase, suggests that phacoemulsification treatment did not modify nucleic acid sequences, which was in line with previous studies [27]. and a display, allowing the surgeon to be far from the ocular surface (see upper left inset). On the right, a surgical display reproduces the operating field in real time. This viewing system improves both the safety and efficiency of cataract surgery during the COVID-19 pandemic

This surgical procedure for eye mock-up requires some considerations. First, the use of an ssRNA probe diluted in saline does not consider both human chamber resistance (artificial structure vs. human anterior chamber) and human fluid composition (saline solution vs. aqueous one). Otherwise, open-globe injuries can be well represented by this model. Second, ssRNA concentrations might not tightly reproduce the minimal virus load that might occur in the human-infected anterior chamber, although five ssRNA dilutions were tested in these studies. Furthermore, the expression of endogenous RNAse (endonuclease, EDN) in human mucosal tissues and biological fluids as natural innate immune defenses might decrease the local and likewise aerosolized "viral load," although the risk of infection cannot be completely abolished [28]. Finally, the strip position $(15 \mathrm{~cm}$ and not $30 \mathrm{~cm}$ ) over the artificial ocular surface (mock-up) did not exactly reproduce the distance between the operator and the ocular surface. While previous studies demonstrated aerosol delivery at $30 \mathrm{~cm}$, our focus was to verify the delivery of potentially intact filaments [28].

The recent COVID-19 pandemic has led to increasing interest in the fact that aerosol produced during cataract surgery can affect the safety of healthcare operators if a minimal viral RNA load can persist inside cells/tissues/ fluids and be released with droplets/aerosol [29-31]. Although not related to "viral/bacterial load," high-risk infection can occur during phacoemulsification generating of aerosol/droplets containing pathological sequences [32]. 
The use of PPIs for outpatients/inpatients and operating microscopes equipped with 3D screen visualization systems (surgery room; Fig. 3) has received great interest in dental and ocular surgery, thus allowing for performing the activity at a distance and with a face shield without interfering with the routine procedure [33-35].

Since (1) minimal aerosol can potentially reach the operator's face during phacoemulsification, and (2) the absence of virus/bacteria cannot be assured in anterior chamber of any patient undergoing phacoemulsification cataract surgery, the ability of intact ssRNA to be projected at short distances is possible.

These findings coupled with frontline operators' experiences aim to increase attention regarding creation of best practices in order to avoid potential transmission of RNA viruses, particularly in the field of surgical ophthalmology [36].

Acknowledgements We thank Fondazione Roma (Rome, Italy) for continuous support. Many thanks to Dr. Giuliana Facciolo and Dr. Francesca Petruzzella (IRCCS-Fondazione Bietti) for technical assistance during experimental phacoemulsification cataract.

Author contributions AC, FS, AM: conceived and wrote the manuscript; AC, FS, PC, GR: performed the experimental phacoemulsification; BOB, LB, CC, AR, AM: performed pre-analytical and analytical analysis; all approved the final version of the manuscript.

Funding Italian Ministry of Health provided financial support in the form of Ricerca Corrente (RC2765949) funding. The sponsor had no role in the design or conduct of this research.

Data availability All data are in the manuscript.

Code availability $\mathrm{n} / \mathrm{a}$.

\section{Declarations}

Ethics approval For this type of study, formal consent is not required. This article does not contain any studies with human participants performed by any of the authors.

\section{Consent to participate $\mathrm{n} / \mathrm{a}$.}

Consent for publication See form.

Conflict of interest The authors declare no competing interests.

\section{References}

1. Guo YR, Cao QD, Hong ZS, Tan YY, Chen SD, Jin HJ, Tan KS, Wang DY, Yan Y (2020) The origin, transmission and clinical therapies on coronavirus disease 2019 (COVID-19) outbreakan update on the status. Mil Med Res 7(1):11. https://doi.org/10. 1186/s40779-020-00240-0
2. Wang MY, Zhao R, Gao LJ, Gao XF, Wang DP, Cao JM (2020) SARS-CoV-2: structure, biology, and structure-based therapeutics development. Front Cell Infect Microbiol 10:587269. https://doi. org/10.3389/fcimb.2020.587269

3. Cafiero C, Re A, Micera A, Palmirotta R, Monaco D, Romano F, Fabrizio C, Di Francia R, Cacciamani A, Surico PL, D'Amato G, Pisconti S (2020) Pharmacogenomics and pharmacogenetics: in silico prediction of drug effects in treatments for novel coronavirus SARS-CoV2 disease. Pharmgenomics Pers Med 13:463-484. https://doi.org/10.2147/PGPM.S270069

4. Tran K, Cimon K, Severn M, Pessoa-Silva CL, Conly J (2012) Aerosol generating procedures and risk of transmission of acute respiratory infections to healthcare workers: a systematic review. PLoS ONE 7:e35797. https://doi.org/10.1371/journal.pone.00357 97

5. Fontes D, Reyes J, Ahmed K, Kinzel M (1994) (2020) A study of fluid dynamics and human physiology factors driving droplet dispersion from a human sneeze. Phys Fluids 32(11):111904. https:// doi.org/10.1063/5.0032006

6. Meselson M (2020) Droplets and Aerosols in the Transmission of SARS-CoV-2. N Engl J Med 382:2063. https://doi.org/10.1056/ NEJMc2009324

7. Wu P, Duan F, Luo C, Liu Q, Qu X, Liang L, Wu K (2020) Characteristics of ocular findings of patients with coronavirus disease 2019 (COVID-19) in Hubei Province, China. JAMA Ophthalmol 138:575-578. https://doi.org/10.1001/jamaophthalmol.2020.1291

8. Xia J, Tong J, Liu M, Shen Y, Guo D (2020) Evaluation of coronavirus in tears and conjunctival secretions of patients with SARSCoV-2 infection. J Med Virol 92:589-594. https://doi.org/10. $1002 / j m v .25725$

9. Leonardi A, Rosani U, Brun P (2020) Ocular surface expression of SARS-CoV-2 receptors. Ocul Immunol Inflamm 28:735-738. https://doi.org/10.1080/09273948.2020.1772314

10. Jarrom D, Elston L, Washington J, Prettyjohns M, Cann K, Myles S, Groves P (2020) Effectiveness of tests to detect the presence of SARS-CoV-2 virus, and antibodies to SARS-CoV-2, to inform COVID-19 diagnosis: a rapid systematic review. BMJ Evid Based Med. https://doi.org/10.1136/bmjebm-2020-111511

11. Goncharova EA, Dedkov VG, Dolgova AS, Kassirov IS, Safonova MV, Voytsekhovskaya Y, Totolian AA (2020) One-step quantitative RT-PCR assay with armored RNA controls for detection of SARS-CoV-2. J Med Virol. https://doi.org/10.1002/jmv.26540.10. 1002/jmv. 26540

12. Dortet L, Ronat JB, Vauloup-Fellous C, Langendorf C, Mendels DA, Emeraud C, Oueslati S, Girlich D, Chauvin A, Afdjei A, Bernabeu S, Le Pape S, Kallala R, Rochard A, Verstuyft C, Fortineau N, Roque-Afonso AM, Naas T (2020) Evaluating ten commercially-available SARS-CoV-2 rapid serological tests using the STARD (standards for reporting of diagnostic accuracy studies) method. J Clin Microbiol. https://doi.org/10.1128/JCM.02342-20

13. McGhee CNJ, Dean S, Freundlich SEN, Gokul A, Ziaei M, Patel DV, Niederer RL, Danesh-Meyer HV (2020) Microdroplet and spatter contamination during phacoemulsification cataract surgery in the era of COVID-19. Clin Exp Ophthalmol 48:1168-1174. https://doi.org/10.1111/ceo.13861

14. Shetty N, Kaweri L, Khamar P, Balakrishnan N, Rasheed A, Kabi P, Basu S, Shetty R, Nuijts R, Sinha RA (2020) Propensity and quantification of aerosol and droplet creation during phacoemulsification with high-speed shadowgraphy amid COVID-19 pandemic. J Cataract Refract Surg 46:1297-1301. https://doi.org/10. 1097/j.jcrs.0000000000000289

15. Naveed H, May Chew F, Lee H, Hughes E, Nanavaty MA (2020) Aerosol generation through pars plana vitrectomy. Br J Ophthalmol. https://doi.org/10.1136/bjophthalmol-2020-317214 
16. Wong R, Bannerjee P, Kumaran N (2020) Aerosol generating procedures in intraocular surgery. Eye (Lond). https://doi.org/10. 1038/s41433-020-0997-7

17. Plemel DJA, Tong CM, Chan SM (2020) Creating aerosolized droplets in phacoemulsification. Can J Ophthalmol. https://doi. org/10.1016/j.jcjo.2020.09.013

18. Rai AS, Mele R, Rai AS, Braga-Mele R (2020) Addressing the concerns of aerosolization during phacoemulsification due to COVID-19: human cadaveric eye with trypan blue. J Cataract Refract Surg. https://doi.org/10.1097/j.jcrs.0000000000000314

19. van Doremalen N, Bushmaker T, Morris DH, Holbrook MG, Gamble A, Williamson BN, Tamin A, Harcourt JL, Thornburg NJ, Gerber SI, Lloyd-Smith JO, de Wit E, Munster VJ (2020) Aerosol and surface stability of SARS-CoV-2 as compared with SARS-CoV-1. N Engl J Med 382:1564-1567. https://doi.org/10. 1056/NEJMc2004973

20. Nagesh D, Ghosh S (2017) A time period study on the efficiency of luminol in the detection of bloodstains concealed by paint on different surfaces. Forensic Sci Int 275:1-7

21. Liyanage S, Ramasamy P, Elhaddad O, Darcy K, Hudson A, Keller J (2020) Assessing visible aerosol generation during vitrectomy in the era of Covid-19. Eye (Lond) 25:1-4. https://doi.org/ 10.1038/s41433-020-1052-4

22. Wong R, Banerjee PJ, Kumaran N (2020) Part 2 model eye simulation: aerosol generating procedures in intraocular surgery. Eye (Lond) 6:1-2. https://doi.org/10.1038/s41433-020-1068-9

23. Noureddin GS, Iovieno A, Eslami M, Weaver T, Meadows H, Yeung SN (2020) Quantification of aerosol generation during cataract surgery. J Cataract Refract Surg. https://doi.org/10.1097/j. jcrs.0000000000000530

24. Iovino C, Caporossi T, Peiretti E (2020) Vitreoretinal surgery tip and tricks in the era of COVID-19. Graefes Arch Clin Exp Ophthalmol 258:2869-2870. https://doi.org/10.1007/ s00417-020-04800-x

25. Koshy ZR, Dickie D (2020) Aerosol generation from high speed ophthalmic instrumentation and the risk of contamination from SARS COVID19. Eye (Lond) 34:1954-1955. https://doi.org/10. 1038/s41433-020-1000-3

26. Wan KH, Huang SS, Young AL, Lam DSC (2020) Precautionary measures needed for ophthalmologists during pandemic of the coronavirus disease 2019 (COVID-19). Acta Ophthalmol 98:221-222. https://doi.org/10.1111/aos.14438

27. Banerjee A, Czinn SJ, Reiter RJ, Blanchard TG (2020) Crosstalk between endoplasmic reticulum stress and anti-viral activities: a novel therapeutic target for COVID-19. Life Sci 255:117842. https://doi.org/10.1016/j.lfs.2020.117842
28. Nissen K, Krambrich J, Akaberi D, Hoffman T, Ling J, Lundkvist A, Svensson L, Salaneck E (2020) Long-distance airborne dispersal of SARS-CoV-2 in COVID-19 wards. Sci Rep 10:19589. https://doi.org/10.1038/s41598-020-76442-2

29. Mohan SV, Hemalatha M, Kopperi H, Ranjith I, Kumar AK (2021) SARS-CoV-2 in environmental perspective: occurrence, persistence, surveillance, inactivation and challenges. Chem Eng J 405:126893. https://doi.org/10.1016/j.cej.2020.126893

30. Elrashdy F, Redwan EM, Uversky VN (2020) Why COVID-19 transmission is more efficient and aggressive than viral transmission in previous coronavirus epidemics? Biomolecules 10:1312. https://doi.org/10.3390/biom10091312

31. Kaur S, Kopsachilis N, Zia RJ (2020) Aerosol generation during phacoemulsification in live patient cataract surgery environment. Cataract Refract Surg. https://doi.org/10.1097/j.jcrs.0000000000 000510

32. Lam PTH, Cheng ACO, Rao S, Lam DSC (2007) Retention of silicone oil droplets from ophthalmic viscosurgical devices in the drainage angle of 2 eyes. J Cataract Refrct Surg 33:1994-1996. https://doi.org/10.1016/j.jcrs.2007.07.011

33. Aweidah H, Safadi K, Jotkowitz A, Chowers I, Levy J (2020) Hybrid telehealth medical retina clinic due to provider exposure and quarantine during COVID-19 pandemic. Clin Ophthalmol 14:3421-3426. https://doi.org/10.2147/OPTH.S276276

34. Asena L, Altınörs DD, Oto S (2020) Surgical microscope drape used as a tent over patient to prevent aerosol dissemination during phacoemulsification. J Cataract Refract Surg. https://doi.org/10. 1097/j.jcrs.0000000000000403

35. Darcy K, Elhaddad O, Achiron A, Keller J, Leadbetter D, Tole D, Liyanage S (2020) Reducing visible aerosol generation during phacoemulsification in the era of Covid-19. Eye (London). https:// doi.org/10.1038/s41433-020-1053-3

36. Chandra A, Haynes R, Burdon M, Laidlaw A, Neffendorf J, Eames I, daCruz L, Lee RW, Charles S, Wilson P, Dick A, Flanagan D, Yorston D, Hingorani M, Wickham L (2020) Personal protective equipment (PPE) for vitreoretinal surgery during COVID-19. Eye 34:1196-1199. https://doi.org/10.1038/s41433-020-0948-3

Publisher's note Springer Nature remains neutral with regard to jurisdictional claims in published maps and institutional affiliations. 